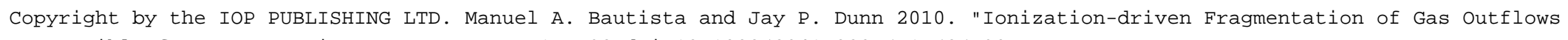
Responsible for FeLoBALs in Quasars," ApJ 717 L98 doi:10.1088/2041-8205/717/2/L98

The Astrophysical Journal LetTers, 717:L98-L102, 2010 July 10

doi:10.1088/2041-8205/717/2/L98

(c) 2010. The American Astronomical Society. All rights reserved. Printed in the U.S.A.

\title{
IONIZATION-DRIVEN FRAGMENTATION OF GAS OUTFLOWS RESPONSIBLE FOR FeLoBALs IN QUASARS
}

\author{
Manuel A. Bautista ${ }^{1,2}$ And Jay P. DunN ${ }^{2}$ \\ ${ }^{1}$ Department of Physics, Western Michigan University, Kalamazoo, MI 49008-5252, USA \\ ${ }^{2}$ Department of Physics, Virginia Polytechnic and State University, Blacksburg, VA 24061, USA \\ Received 2010 February 23; accepted 2010 June 4; published 2010 June 21
}

\begin{abstract}
We show that time variations in the UV ionizing continuum of quasars, on scales of $\sim 1 \mathrm{yr}$, affect the dynamic structure of the plasmas responsible for low-ionization broad absorption lines. Variations of the ionizing continuum produce non-equilibrium photoionization conditions over a significant fraction of the absorbing clouds and supersonically moving ionization fronts. When the flux drops, the contraction of the ionized region drives a supersonic cooling front toward the radiation source and a rarefaction wave in the opposite direction. The pressure imbalance is compensated by an increased speed of the cool gas relative to the front. When the flux recovers, the cool gas is re-ionized and re-heated by a supersonic ionization front traveling away from the radiation source and a forward shock is created. The re-heated clouds equilibrate to a temperature of $\sim 10^{4} \mathrm{~K}$ and are observed to have different radial velocities than the main cloud. Such fragmentation seems consistent with the multicomponent structure of troughs seen in some objects. The velocity differences measured among various components in the quasars QSO 2359-1241 and SDSS J0318-0600 can be reproduced by our model if strong magnetic fields $(\sim 10 \mathrm{mG})$ are present within the clouds.
\end{abstract}

Key words: galaxies: active - hydrodynamics - quasars: absorption lines - shock waves

Online-only material: color figure

\section{INTRODUCTION}

Gas outflows are a potential source of feedback that regulates the evolution of the central black hole, the host galaxy, and the intergalactic medium (e.g., Elvis 2006). In the restframe UV spectra of 10\%-20\% of all quasars (Knigge et al. 2008; Foltz et al. 1987), outflows are manifested in blueshifted broad absorption lines (BALs). The most common of these are associated with resonance lines of medium-ionization species, such as C IV, N v, O vi, Si IV, and $\mathrm{HI}$, and can reach velocities as high as 50,000 $\mathrm{km} \mathrm{s}^{-1}$ (Weymann 1995; Turnshek 1995). Unfortunately, their saturated nature limits their utility in determining the physical characteristics of the outflow. A subset of BAL quasars, known as LoBALs, also shows complexes of narrower (on the order of $\sim 100 \mathrm{~km} \mathrm{~s}^{-1}$ ) absorption features from low-ionization species such as $\mathrm{Mg}$ II, Al II, and Si II. Among these, objects that include troughs of metastable Fe II or Fe III are specifically called FeLoBALs. These are useful because the column densities of metastable levels offer valuable diagnostics of the outflow (Gabel et al. 2006; Arav et al. 2005, and references therein). These studies place the gas responsible for the FeLoBAL at distances between $\sim 1 \mathrm{kpc}$ (Korista et al. 2008; Moe et al. 2009) and up to $\sim 10 \mathrm{kpc}$ (Dunn et al. 2010).

We studied the various kinematic components in the FeLoBAL troughs of two quasars, QSO 2359-1241 and SDSS $\mathrm{J} 0318-0600, z=0.868$ and 1.967, respectively (Bautista et al. 2010). It was found that among the various kinematic components, the highest column density component of each system also had the highest particle density, while all other components have densities of about one-quarter of that of the main component. Moreover, all of the components seem to be located at the same distance from the central source, suggesting that they are all related to each other. This is also consistent with the finding of Voit et al. (1993) that BALs and LoBALs could be simultaneously explained by the ablation of either a single large cloud or many scattered clouds.
Another characteristic of FeLoBALs is that, within the sample of known objects, they are borderline density bounded. FeLoBAL quasars are seen in absorption from species that arise from the ionization front (IF) of the cloud (e.g., Si II and Fe II), while atoms from a neutral cold region, e.g., C I or Fe I, are not seen. This seems to point out some relationship between the IF and the nature of the clouds. The location of the IF of a cloud of a given density depends on the flux of hydrogen ionizing photons, particularly in the $\sim 1-4$ Ryd energy range, from the radiation source. However, quasar continua are known to vary in the optical and UV within timescales on the order of one year (Kaspi et al. 2000). Hence, it is important to understand the effect of the varying radiation field on the structure of the FeLoBAL.

In this Letter, we show that when the flux that ionizes a FeLoBAL cloud drops, the depth of the ionized region often shrinks at supersonic speeds followed by a cooling front, as explained in Sections 2 and 3. In Section 4 we show that the supersonic cooling front creates a rarefaction wave, traveling in the opposite direction to the front, which results in the fragmentation of the cloud. Under some conditions, such a fragmentation can explain the observed structure of FeLoBALs.

\section{MAGNITUDE SCALES AND THE EFFECT OF FLUX VARIATIONS ON FeLoBALs}

Let us start with the simple picture illustrated in Figure 1. Here, $r$ is the depth of the neutral region of the cloud and is measured from the far end of the cloud toward the central source. For a given luminosity of the source and a cloud of constant particle density $n_{H}$ moving in a medium of negligible density, one can draw an imaginary radius within which the cloud is fully ionized. This is

$$
R_{\mathrm{if}}\left(Q_{H}, n_{H}, l\right)=\left(\frac{Q_{H}}{4 \pi n_{H}^{2} \alpha l}\right)^{1 / 2},
$$




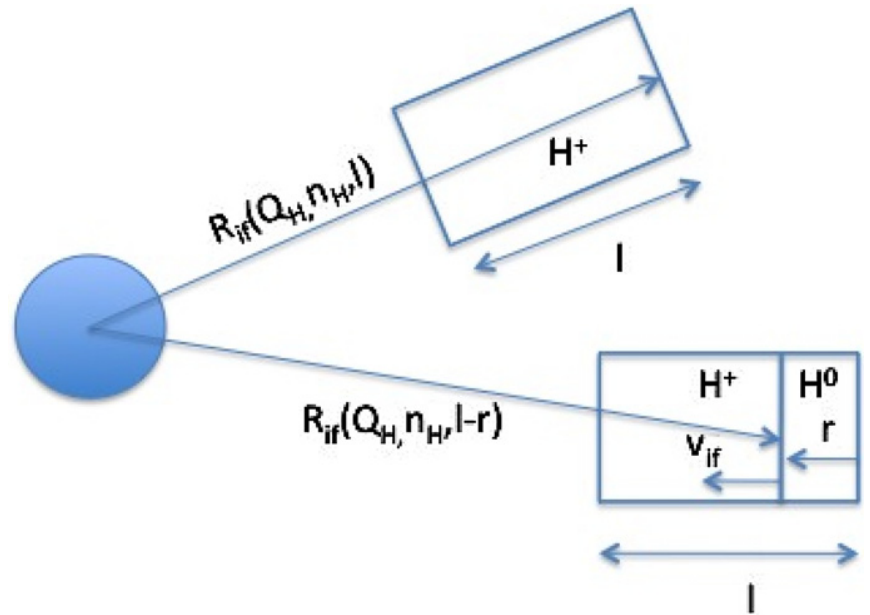

Figure 1. Illustration of a photoionized cloud of depth $l$ traveling away from the light source. The upper cloud is right at $R_{\mathrm{if}}$ and the whole depth $l$ is ionized. In the lower cloud, only a depth $l-r$ is ionized due to either a larger physical distance or a drop in $Q_{H}$.

(A color version of this figure is available in the online journal.)

where $Q_{H}$ is the rate of hydrogen ionizing photons from the central source, $\alpha$ is the recombination rate coefficient, and $l$ is the depth of the cloud. For a cloud located beyond $R_{\mathrm{if}}$, the ionized depth $(l-r)$ is related to the physical distance $(R)$ as $R^{2}(l-r)=Q_{H} /\left(4 \pi n_{H}^{2} \alpha\right)$. Thus, FeLoBAL outflows that result from the formation of an IF, but without an extended neutral region, must be located right at $R_{\mathrm{if}}$. Then, a drop $\Delta Q_{H}$ in the ionization flux, i.e., $Q_{H}^{\prime}=Q_{H}-\Delta Q_{H}$, will create a region of depth $r=l \times \Delta Q_{H} / Q_{H}$ of either neutral or recombining plasma. Here, $l \approx N_{H} / n_{H}$, where $N_{H}$ is the total column density of the cloud and has a typical value of $\sim 10^{20.5} \mathrm{~cm}^{-2}$. In deriving this equation, we assumed that $l \ll R_{\mathrm{if}}$ and $r \lll$ In QSO 2359-1241 and SDSS J0318-0600, $n_{H}$ are $\sim 10^{4.4} \mathrm{~cm}^{-3}$ and $\sim 10^{3.4} \mathrm{~cm}^{-3}$ and the sizes of the absorbers are $10^{16}-10^{17} \mathrm{~cm}$ (i.e., $\sim 0.01-0.1$ light years). The change in the ionization of the gas is not instantaneous, but delayed by the recombination timescale of the plasma $\tau_{\alpha}=1 / n_{H} \alpha$, with $\alpha \approx 2 \times 10^{-13} \mathrm{~s}^{-1} \mathrm{~cm}^{-3}$ at $T_{e} \approx 10^{4} \mathrm{~K}$. Then, $\tau_{\alpha} \approx 10 \mathrm{yr}$ in QSO 2359-1241 and $100 \mathrm{yr}$ in SDSS J0318-0600. For example, in the case that $Q_{H}$ is abruptly shut off, the size of the ionized region is

$$
l-r(t)=l e^{-t / \tau_{\alpha}},
$$

where $t$ is the time measured from the moment that the flux is turned off. Thus, the depth of the ionized region shrinks with a speed

$$
v_{\mathrm{if}}=\frac{d(l-r(t))}{d t} \approx \frac{l}{\tau_{\alpha}} \text { for } t \ll \tau_{\alpha} .
$$

With the values of $l$ and $\tau_{\alpha}$ derived above, $v_{\text {if }} \approx N_{H} \alpha \approx$ $600 \mathrm{~km} \mathrm{~s}^{-1}$, which is much faster than the speed of sound in the plasma.

The width of quasi-static IFs is on the order of the mean free path for ionizing radiation, i.e., $1 / n_{H} \sigma_{H}$, where $\sigma_{H} \approx$ $6 \times 10^{-18} \mathrm{~cm}^{2}$ is the photoionization cross section. Thus, the IFs in QSO 2359-1241 and SDSS J0318-0600 are on the order of $10^{13}-10^{14} \mathrm{~cm}$, which are much smaller than the distance on which the IF retreats for flux variations on $\sim 1 \mathrm{yr}$ timescale. Still, these continuum variations, which are much shorter than the recombination time, will broaden the IF, creating an extended recombining region. But, as the IF departs from ionization equilibrium it also departs from thermal equilibrium. In a static
IF, the temperature drops to a much lower value for a given value of the optical depth to heating photons. The optical depth is

$$
\tau=\sigma_{H} \int_{0}^{S_{\max }} n_{H^{0}} d s^{\prime},
$$

with $n_{H^{0}}$ the neutral hydrogen density. Let us assume, for instance, a static IF of the form $n_{H^{0}}=n_{H} \times\left(1-e^{-s / \delta}\right)$. As the ionized region shrinks, the characteristic depth of the front increases to $\delta^{\prime}>\delta$. At an early time $t$ after an instantaneous retreat of the IF, $n_{H^{0}} \approx t / t_{\alpha}$. Thus, for an IF broadening by a factor $v$ it will take a time $t \approx t / v$ for the cooling front to start moving with the retreating IF. Then, if the value of $\tau$ for which the temperature drop remains constant, the depth within the IF at which this happens, $S_{\max }$, will be reduced with respect to $\delta^{\prime}$. For example, if the change in temperature occurred at $S_{\max } / \delta=1$ in the static IF, $S_{\max } / \delta^{\prime}=0.68$ for $\delta^{\prime} / \delta=2, S_{\max } / \delta^{\prime}=0.41$ for $\delta^{\prime} / \delta=5$, and $S_{\max } / \delta^{\prime}=0.28$ for $\delta^{\prime} / \delta=10$. In the region of new lower equilibrium temperature cooling of the plasma proceeds much faster than recombination, because the cooling timescale is only $\tau_{T} \approx 2 \times 10^{4} / n_{H} \operatorname{yr}$ (Spitzer 1998, p. 139). In conclusion, the drop in temperature closely follows the supersonic shrinking of the ionized region despite the broadening IF. Note that a drop in temperature makes recombination speed up, because $\alpha$ increases as $T_{e}^{-1 / 2}$ as the electron temperature $T_{e}$ decreases.

What happens when the flux increases again? As the ionizing flux rises, the neutral fragment of the cloud will be re-ionized. Thus, an IF will travel through the neutral fragment, in the direction away from the ionizing source. This process is governed by the equation

$$
\frac{\Delta Q_{H}}{4 \pi R^{2}}=n_{H^{0}} v_{\mathrm{IF}} .
$$

The speed of the IF in this case is proportional to the change in radiative flux and can be highly supersonic, in which case it will send shocks ahead of the IF and rarefaction waves in the opposite direction.

In conclusion, time variations of the ionizing flux are expected to have two kinds of effects. (1) In the region neighboring the IF, the ionization varies with time and departure from photoionization equilibrium could occur. This effect is out of the scope of this Letter, but will be the subject of future investigation. (2) The varying ionizing flux will create supersonic cooling and heating fronts traveling through the ionized fraction of the cloud and the neutral region. These will be accompanied by rarefaction waves and shocks. This process is investigated in Section 4 of this Letter, but first we will derive a more accurate description of the dynamics of the IF.

\section{DYNAMICS OF IONIZATION FRONTS WITHIN OUTFLOWING CLOUDS}

The IF within a cloud is defined as the region where the ionization drops to near zero. Thus, we solve for $n_{e}$ in the timedependent photoionization equation

$$
\frac{d n_{e}}{d t}+\nabla \cdot\left(n_{e} u\right)=\Gamma-\Lambda,
$$

where $t$ is the time, $u$ is the velocity of the gas, and $\Gamma$ and $\Lambda$ are the ionization and recombination rates per unit volume,

$$
\Gamma=-\nabla \cdot \Phi_{H}=-\nabla \cdot \frac{Q_{H} e^{-\tau(x)}}{4 \pi R^{2}},
$$




$$
\Lambda=\alpha_{H} n_{e}^{2},
$$

where $\tau(x)$ is the optical depth within the cloud up to the depth $x$.

Integrating Equation (3) in the co-moving frame of the IF ( $u=0$ ) over the ionized volume $V=\Omega R^{2}(l-r)$, with $\Omega$ the covering solid angle, one finds a differential equation for $r(t)$ :

$$
\frac{d(l-r(t))}{d t}=\frac{Q_{H}}{4 \pi n_{e} R^{2}}-\frac{l-r(t)}{\tau_{\alpha}} .
$$

For gas clouds much smaller than the distance to the ionizing source, i.e., $l \ll R$, one can assume constant density during a crossing time of the cloud $(\sim l / v)$. Then

$$
l-r(t)=l e^{-t / \tau_{\alpha}}\left[\frac{1}{\tau_{\alpha} Q_{H}(0)} \int_{0}^{t} Q_{H}\left(t^{\prime}\right) e^{t^{\prime} / \tau_{\alpha}} d t^{\prime}+1\right] .
$$

To understand this solution, it is useful to check a couple of cases

1. $Q_{H}(t)$ varying as a step function. Let us assume that at $t=0$ the absorber just reached $R_{\mathrm{if}}$ when the continuum flux was $Q_{0}$. Thus, the cloud is fully ionized and a drop in $Q_{H}$ will produce an IF. If the absorber were closer than $R_{\mathrm{if}}$, then it could remain fully ionized even when $Q_{H}$ is reduced. If the flux drops to $\xi Q_{0}$ at $t=0$ for $t>0$, one gets

$$
l-r(t)=l \times\left[\xi+(1-\xi) e^{-t / \tau_{\alpha}}\right]
$$

and

$$
v_{\mathrm{IF}}=(1-\xi) \frac{l}{\tau_{\alpha}} e^{-t / \tau_{\alpha}},
$$

which is consistent with the result of Equation (4) and yields supersonic $v_{\mathrm{IF}}$ for flux variations of $\sim 5 \%$ or greater, i.e., $\xi \leqslant 0.95$.

2. $Q_{H}(t)$ from observed quasar continua. Here we compute the ionization of the cloud versus time for selected quasar continua observed by Kaspi et al. (2000) using Equation (10). Figure 2 shows the observed flux, the IF location with respect to the total depth of the cloud $l$, and $v_{\mathrm{IF}}$ for three quasars. These objects are chosen out of a sample of 17 quasars observed by Kaspi et al. because these illustrate the types of dynamical behaviors of the IF. Because the calculations are done for illustrative purposes only, we disregard the uncertainties on the measured fluxes. Note, for example, that in PG0844 the $r(t) / l$ ratio is generally decreasing with time because the average ionizing continuum is lower than at the initial time of observation. This overall behavior is somewhat arbitrary as it depends on the initial observation. What is more meaningful, though, is the instantaneous velocity of the IF front, computed as $\Delta r / \Delta t$, and this is seen to reach highly supersonic values. This is because the flux levels in PG0844, PG1226, and PG1411 vary by $\sim 40 \%, \sim 50 \%$, and $\sim 30 \%$ with respect to the value at $t=0$ in less than a year. Clearly, this determination of velocities is sensitive to the observational errors as well as the sampling of the quasar luminosity. Hence, we make no definitive claims on specific objects. However, the prediction that highly supersonic fronts are expected is supported in every object of the available sample.

\section{SUPERSONIC IONIZATION FRONTS AND COOLING FRONTS}

The supersonic IF is accompanied by a cooling front that leaves a pressure step behind. This creates a rearward-facing, i.e., in the opposite direction to the IF, rarefaction wave and a forward-facing shock (Strachan \& Ahlborn 1975; Ahlborn \& Strachan 1973). Under homogeneous conditions and for an infinite flow stream these waves and shocks will form periodically (Schnerr \& Adam 1997). As discussed before, the fragments once created become re-ionized and re-heated when the flux recovers. Then one can study the jump conditions between the main cloud and the fragments assuming constant temperature.

In one dimension, the jump conditions across the rarefaction wave are

$$
\begin{aligned}
\rho_{1} w_{1} & =\rho_{2} w_{2}, \\
\rho_{1} w_{1}^{2}+P_{1}+\frac{B_{1}^{2}}{8 \pi} & =\rho_{2} w_{2}^{2}+P_{2}+\frac{B_{2}^{2}}{8 \pi}, \\
B_{1} w_{1} & =B_{2} w_{2},
\end{aligned}
$$

where $\rho_{i}$ is the mass density of the gas, $w$ is the bulk velocity of the gas in the co-moving frame of the wave $\left(w=v-v_{\text {wave }}\right)$, $P$ is the thermal pressure, and $B$ is the strength of the magnetic field. Because the jump is essentially isothermal we adopt $a^{2}=P_{1} / \rho_{1}=P_{2} / \rho_{2}$, and from Equations (13)-(15) one gets

$$
w_{1}^{2}=\left(\frac{\rho_{2}}{\rho_{1}}\right)\left[a^{2}+u_{A 1}^{2}\left(\frac{\rho_{2}}{\rho_{1}}+1\right)\right],
$$

where $u_{A 1}=B_{1} /\left(8 \pi \rho_{1}\right)^{1 / 2}$ is the Alfvén velocity. Thus, the difference of velocities between the fragments and the main cloud is

$v_{2}-v_{1}=w_{2}-w_{1} \approx \pm 20 \times\left[1+\frac{5}{4}\left(\frac{v_{A 1}}{13 \mathrm{~km} \mathrm{~s}^{-1}}\right)^{2}\right]^{1 / 2} \mathrm{~km} \mathrm{~s}^{-1}$,

where we have used typical conditions $\rho_{1} / \rho_{2}=4$ and $a \approx$ $13 \mathrm{~km} \mathrm{~s}^{-1}$. This result shows that rarefaction waves, originated by supersonic IF, yield lower density fragments that detach from the main cloud with relative velocities of tens of $\mathrm{km} \mathrm{s}^{-1}$ in the absence of magnetic fields and much larger velocities when these are present.

There is evidence suggesting the presence of magnetic fields in BALs. Large trough widths, on the order of $100 \mathrm{~km} \mathrm{~s}^{-1}$, are observed in FeLoBALs, with the largest widths being generally associated with the main absorber (see Table 1 of Bautista et al. 2010). The widths are much greater than expected from thermal broadening and seem to indicate supersonic turbulent velocities which could only be sustained by equally fast Alfvén waves. The alternative scenario, that observed trough widths could be mostly due to bulk velocity gradients along the line of sight, is inconsistent with the fact that clouds have been able to travel $\sim 1-10 \mathrm{kpc}$ away from the active galactic nucleus (AGN) without diffusing into the interstellar medium. Moreover, it is difficult to understand how bulk velocity gradients could yield similar trough profiles for ions with different ionization stages (but see Arav et al. 1999).

Strong magnetic fields in BAL clouds have been proposed in the past. de Kool \& Begelman (1995) pointed out that magnetic pressures in BAL clouds must exceed thermal and 

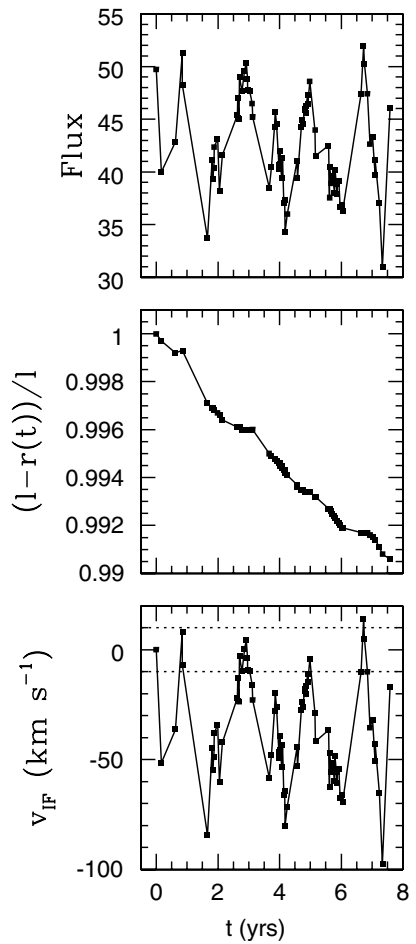
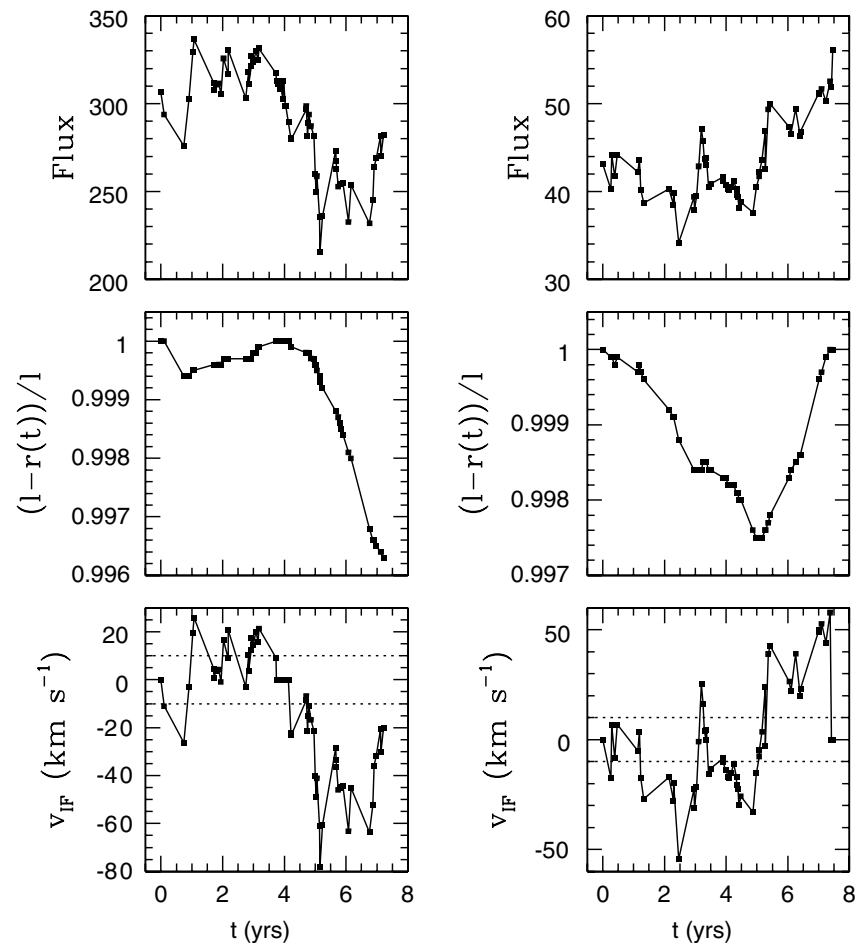

Figure 2. Flux, $r(t)$, and $v_{\mathrm{IF}}(t)$ for a sample of PG quasars observed by Kaspi et al. (2000). The results are for PG0844 (left panels), PG1226 (middle panels), and PG1411 (right panels). The calculations assume $\tau_{\alpha}=120 \mathrm{yr}$ and $l=10^{17} \mathrm{~cm}$. The horizontal dotted lines in the $v_{\mathrm{IF}}$ vs. $t$ plots delimit the approximate range for subsonic motion.

radiative pressures to confine the clouds when accelerated up to thousands of $\mathrm{km} \mathrm{s}^{-1}$. Their models for acceleration of BAL clouds yield magnetic field strengths on the order of $10 \mathrm{mG}$. Further, Furlanetto \& Loeb (2001) argue that BAL quasar outflows could carry a large fraction of the magnetic energy of the intergalactic medium in clusters of galaxies. The source of such magnetic fields could be either from the accretion disk of the AGN (de Kool \& Begelman 1995) or from dynamo-like effects in the plasmas such as in the solar winds and supernova remnants (Giacalone \& Jokipii 2007; Kim \& Balsara 2006; Balsara \& Kim 2005).

We find that magnetic fields on the order of $10 \mathrm{mG}$, i.e., Alfvén velocities of a few hundred $\mathrm{km} \mathrm{s}^{-1}$, would explain the observed velocity separations between the main and the minor components as observed in QSO 2359-1241 and SDSS J0318-0600 (see Bautista et al. 2010).

Another interesting aspect of Equation (17) is that it predicts fragments that are faster than the main cloud as well as fragments that are slower than the main cloud, as seen in FeLoBALs. For example, since $w_{2}=4 \times w_{1}$ if $w_{2}-w_{1}=+300 \mathrm{~km} \mathrm{~s}^{-1}$ and $v_{\text {wave }}=1000 \mathrm{~km} \mathrm{~s}^{-1}$, then $v_{1}=1100 \mathrm{~km} \mathrm{~s}^{-1}$ and $v_{2}=1400 \mathrm{~km} \mathrm{~s}^{-1}$. By contrast, if $w_{2}-w_{1}=-300 \mathrm{~km} \mathrm{~s}^{-1}$ then $v_{1}=900 \mathrm{~km} \mathrm{~s}^{-1}$ and $v_{2}=600 \mathrm{~km} \mathrm{~s}^{-1}$. The faster clouds, in the rest frame of the quasar, are expected to outrun the main cloud and remain undisturbed by it. The survival of the slower clouds depends on the geometry of the problem. In one-dimensional geometry, the slow clouds would be swept up by the main cloud. In two and three dimensions, it is possible that magnetic fields produce surface tension in the clouds capable of withstanding the fluid bulk pressure. The deceleration of the flow along the axis of travel of the main cloud and its fragments yields a bow shock, where the supersonic gas of the main cloud flows smoothly around the small fragment (see Shu 1992).

\section{DISCUSSION AND CONCLUSIONS}

We studied the effects of quasar time variable UV flux on FeLoBAL clouds. In these systems, absorption from Si II and Fe II comes from a region just before an IF. This IF is formed within the absorbing cloud when the cloud crosses a distance $R_{\mathrm{if}}$ from the radiation source. At this distance, when the radiation flux drops the ionized region of the cloud will retreat at supersonic speeds. A cooling front will follow the ionized region and the IF will also retreat and broaden. This creates a pressure imbalance across the IF on timescales much shorter than the sound crossing time. Thus, the cooling front yields a rarefaction wave that travels into the low-temperature region. To conserve momentum across the cloud, the large pressure imbalance is compensated by an increased speed of the cold gas relative to the front. Now, when the flux recovers the neutral rarefied region is re-ionized and re-heated by an IF moving away from the ionizing source. This IF can be highly supersonic, driving a shock ahead of it and a rarefaction wave in the opposite direction. The shocked gas forms new clouds and stabilize at a temperature of $\sim 10^{4} \mathrm{~K}$, like the main cloud. These clouds are detached in velocity space from the main cloud and there are rarefaction waves moving between the clouds.

There are two fundamental parameters in determining the speed of the retreating IF. One is the ratio of the depth of the cloud to the recombination rate, $l / \tau_{\alpha}$, which in turn is proportional to the column density of the cloud, $N_{H}$. Thus, high column density clouds can have faster IFs. The other parameter is the ratio of the variability timescale of the ionizing source to the recombination time. Flux variations on scales of $\sim 1 \mathrm{yr}$ are enough for significant effects in FeLoBALs with particle densities of $\sim 10^{4} \mathrm{~cm}^{-3}$, while variability on shorter 
timescales would affect higher density clouds. When the flux drops, broadening of the IF slows the cooling front down such that marginally supersonic IF may not lead to shocks and waves, but faster IFs will.

The thermal pressure imbalance across the IFs alone yields velocities to the fragments on the order of $\sim 20 \mathrm{~km} \mathrm{~s}^{-1}$ with respect to the main cloud. Much higher velocities can be attained if sizable magnetic pressures are considered. Moreover, in two objects for which complete measurements exist, QSO 2359-1241 and SDSS J0318-0600, the observed separation between kinematic components is well reproduced by magnetic field strengths on the order of $\sim 10 \mathrm{mG}$.

Our model predicts that the detached fragments can be either faster or slower than the main cloud, as observed in FeLoBAL spectra. Survival of the slower fragments depends on the geometry of the problem. We propose that the slower fragments may permeate the main cloud if they are clumpy with a small filling factor and/or if the magnetic surface tension of the clouds is large enough to make the clouds flow around each other.

The physical effects presented here may not be exclusive to FeLoBALs, but could have implications in a variety of galactic and extragalactic phenomena, for example, in cataclysmic variables, variable stars with circumstellar envelopes such as $\eta$ Carinae, and in the narrow-line regions of the AGN.

We thank Nahum Arav and Kirk Korista for fruitful discussions. We also thank the anonymous referee for important suggestions.

\section{REFERENCES}

Ahlborn, B., \& Strachan, J. D. 1973, Can. J. Phys., 51, 1416

Arav, N., Kaastra, J., Kriss, G. A., Korista, K. T., Gabel, J., \& Proga, D. 2005, ApJ, 620, 665

Arav, N., Korista, K. T., de Kool, M., Junkkarinen, V. T., \& Begelman, M. C. 1999, ApJ, 516, 27

Balsara, D. S., \& Kim, J. 2005, ApJ, 634, 390

Bautista, M. A., Dunn, J. P., Arav, N., Korista, K., Moe, M., \& Benn, C. 2010, ApJ, 713, 25

de Kool, M., \& Begelman, M. C. 1995, ApJ, 455, 448

Dunn, J., et al. 2010, ApJ, 709, 611

Elvis, M. 2006, Mem. S. A. It., 77, 573

Foltz, C. B., Weymann, R. J., Morris, S. L., \& Turnshek, D. A. 1987, ApJ, 347, 450

Furlanetto, S. R., \& Loeb, A. 2001, ApJ, 556, 619

Gabel, J. R., Arav, N., \& Kim, T. 2006, ApJ, 646, 742

Giacalone, J., \& Jokipii, J. R. 2007, ApJ, 663, L41

Kaspi, S., Smith, P. S., Netzer, H., Maoz, D., Jannuzi, B. T., \& Giveon, U. 2000, ApJ, 533, 631

Kim, J., \& Balsara, D. S. 2006, Astron. Nachr., 327, 433

Knigge, C., Scarini, S., Goad, M. R., \& Cottis, C. E. 2008, MNRAS, 386, 1426

Korista, K. T., Bautista, M. A., Arav, N., Moe, M., Constantini, E., \& Benn, C. 2008, ApJ, 688, 108

Moe, M., Arav, N., Bautista, M. A., \& Korista, K. T. 2009, ApJ, 706, 525

Schnerr, G. H., \& Adam, S. 1997, J. Thermal Sci., 6, 171

Shu, F. H. 1992, The Physics of Astrophysics. Volume II: Gas Dynamics (Mill Valley, CA: Univ. Science Books)

Spitzer, L. 1998, Physical Processes in the Interstellar Medium (New York: Wiley-Interscience)

Strachan, J. D., \& Ahlborn, B. 1975, Aust. J. Phys., 28, 395

Turnshek, D. A. 1995, in QSO Absorption Lines, ed. G. Meylan (Berlin: Springer), 223

Voit, G. M., Weymann, R. J., \& Korista, K. T. 1993, ApJ, 413, 95

Weymann, R. J. 1995, in QSO Absorption Lines, ed. G. Meylan (Berlin: Springer), 213 